\title{
Correction to: Results of nimotuzumab and vinorelbine, radiation and re-irradiation for diffuse pontine glioma in childhood
}

\author{
Maura Massimino ${ }^{1}$. Veronica Biassoni ${ }^{1} \cdot$ Rosalba Miceli $^{2}$ - Elisabetta Schiavello ${ }^{1} \cdot$ Monika Warmuth-Metz $^{3}$. \\ Piergiorgio Modena ${ }^{4} \cdot$ Michela Casanova $^{1}$ - Emilia Pecori ${ }^{5} \cdot$ Felice Giangaspero $^{6} \cdot$ Manila Antonelli $^{6}$. \\ Francesca Romana Buttarelli ${ }^{7}$. Paolo Potepan ${ }^{8} \cdot$ Bianca Pollo $^{9} \cdot$ Raffaele Nunziata $^{9} \cdot$ Filippo Spreafico $^{1}$. \\ Marta Podda ${ }^{1}$. Andrea Anichini ${ }^{10}$. Carlo Alfredo Clerici ${ }^{111} \cdot$ Iacopo Sardi $^{12} \cdot$ Loris De Cecco $^{13} \cdot$ Udo Bode $^{14}$. \\ Ferdinand Bach $^{15} \cdot$ Lorenza Gandola $^{5}$
}

Published online: 16 May 2018

(c) Springer Science+Business Media, LLC, part of Springer Nature 2018

\section{Correction to: J Neurooncol (2014) 118:305-312 https://doi.org/10.1007/s11060-014-1428-z}

The therapeutic experience reported in the paper was conceived after the use of nimotuzumab and radiotherapy (BSCPED-05 international multicentric trial, EUDRACT 2005-003100-11) in 2009 when we decided to explore the activity of the same combination plus vinorelbine (see the paper for the rationale).

The company developing nimotuzumab in Europe agreed for its compassionate use in DIPG patients in this context.
The Ethical Committee (EC) of our Institution (Fondazione IRCCS Istituto Nazionale dei Tumori, in Milan) approved any single patient-case admission to the compassionate use of the combination of nimotuzumab with vinorelbine and radiotherapy, according to the procedure designed by the Italian Ministry of Health and by the national Agency for Drug Evaluation (AIFA).

The signed informed consent, approved by the EC, included the possibility of collecting and publishing data, according to the law, in an observational report.

Meanwhile, after some time (February 2012), an ancillary biological study on the same cohort of patients was in fact approved by the Ethical Committee also concerning the analysis of blood samples for biological research.

8 Radiology Unit, Fondazione IRCCS Istituto Nazionale dei Tumori (INT), Via Venezian 1, 20133 Milano, Italy

9 Neuropathology Unit, IRCCS Istituto Neurologico Carlo Besta, Via Celoria, 20133 Milano, Italy

10 Human Tumor Immunobiology Unit, Fondazione IRCCS Istituto Nazionale dei Tumori (INT), Via Venezian 1, 20133 Milano, Italy

11 Physiopathology Department, State Medical School, Via Festa del Perdono, 20100 Milano, Italy

12 Neuro-Oncology Unit, Ospedale Meyer, Viale Pieraccini 24, 50139 Firenze, Italy

13 Molecular Therapies Unit, Fondazione IRCCS Istituto Nazionale dei Tumori (INT), Via Venezian 1, 20133 Milano, Italy

14 Department of Pediatric Hematology/Oncology, University of Bonn Medical School, Bonn, Germany

15 Oncoscience AG, Hafenstraße 32, 22880 Wedel, Germany 
In conclusion, our paper was a report of the clinical data from a cohort of patients treated in the context of a compassionate use approved as such.
As to the paper writing, we adopt a post-hoc analysis as a phase 2 study but we acknowledge as an ERRATUM that this was not a formal phase 2 study. 